\title{
First-in-class HIF2a antagonist safe and effective
}

Poorly regulated angiogenesis is a hallmark of many advanced-stage renal cell carcinomas, and the ability of cancer cells to survive and/or proliferate in hypoxic environments might explain resistance to anti-VEGF targeted therapies. Now, data from a clinical trial provide evidence of the safety and effectiveness of PT2385, a first-in-class hypoxia-inducible factor $2 \alpha$ (HIF2 $\alpha$ ) antagonist, that reduces the expression of VEGF and several other hypoxia-inducible genes.

Researchers conducted a phase II trial with dose-escalation and dose-expansion phases, in a total of 51 patients previously treated with a median of four agents, including VEGF-targeted therapies in all patients. Rapid reductions in serum levels of erythropoietin, a HIF2a target, were observed at all doses, confirming specificity. PT2385 was well tolerated by most patients; the most common grade $\geq 3$ adverse events were anaemia $(10 \%)$, hypoxia $(10 \%)$, lymphopenia (8\%) and hypophosphataemia
(8\%). No patients discontinued treatment owing to adverse events and 13 patients continued to receive treatment for $>1$ year.

PT2835 enabled a disease-control rate of $66 \%$ : one patient ( $2 \%$ ) had a complete response, with partial responses and stable disease observed in $12 \%$ and $52 \%$ of patients, respectively. The researchers highlighted that the $14 \%$ response rate and low risk of serious adverse events compares favourably with the findings of phase II studies using other antiangiogenic agents, confirming a need for further investigation of PT2835. Results from the planned second and third parts of this trial, in which PT2385 will be investigated in combination with nivolumab or cabozantinib, respectively, are eagerly awaited.

Peter Sidaway

ORIGINAL ARTICLE Courtney, K. D. et al. Phase I doseescalation trial of PT2385, a first-in-class hypoxia-inducible factor-2 $\alpha$ antagonist in patients with previously treated advanced clear cell renal cell carcinoma. J. Clin. Oncol. https://doi.org/10.1200/JCO.2017.74.2627 (2017) 